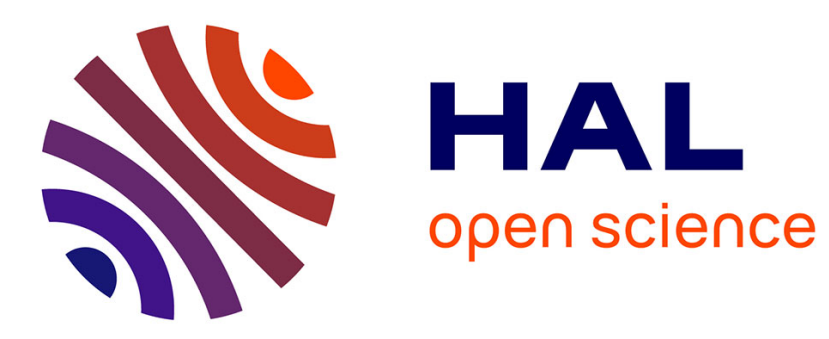

\title{
Computation of shock profiles in radiative hydrodynamics
}

Jean-François Coulombel, Pauline Lafitte

\section{To cite this version:}

Jean-François Coulombel, Pauline Lafitte. Computation of shock profiles in radiative hydrodynamics. Communications in Computational Physics, 2009, 6 (5), pp.1118-1136. 10.1007/s00193-012-0368-9 . hal-00768629

\section{HAL Id: hal-00768629 \\ https://hal.science/hal-00768629}

Submitted on 19 Jul 2018

HAL is a multi-disciplinary open access archive for the deposit and dissemination of scientific research documents, whether they are published or not. The documents may come from teaching and research institutions in France or abroad, or from public or private research centers.
L'archive ouverte pluridisciplinaire HAL, est destinée au dépôt et à la diffusion de documents scientifiques de niveau recherche, publiés ou non, émanant des établissements d'enseignement et de recherche français ou étrangers, des laboratoires publics ou privés. 


\title{
Computation of shock profiles in radiative hydrodynamics
}

\author{
Jean-François Coulombel ${ }^{\dagger}$ and Pauline Lafitte ${ }^{\ddagger}$ \\ $\dagger$ CNRS \\ $\dagger \ddagger$ Project Team SIMPAF INRIA Futurs and Université Lille 1, \\ Laboratoire Paul Painlevé, Cité Scientifique \\ 59655 VILLENEUVE D'ASCQ CEDEX, France \\ Emails: jfcoulom@math.univ-lille1.fr, lafitte@math.univ-lille1.fr
}

July 19, 2018

\begin{abstract}
This article is devoted to the construction of a numerical scheme to solve the equations of radiative hydrodynamics. We use this numerical procedure to compute shock profiles and illustrate some earlier theoretical results about their smoothness and monotonicity properties. We first consider a scalar toy model, then we extend our analysis to a more realistic system for the radiative hydrodynamics that couples the Euler equations and an elliptic equation.
\end{abstract}

\section{Introduction}

The aim of this paper is to construct a numerical scheme to compute shock profiles for some models of radiative hydrodynamics. Such models couple a hydrodynamics part, typically the Euler equations of compressible gas dynamics, with a nonlocal source term. In what follows, the nonlocal operator will be a convolution operator on the real line. Our numerical procedure is based on a splitting strategy. In a first time substep, we solve the hydrodynamics part by means of a conservative low-diffusive scheme (either the Godunov scheme or the so-called Lagrange-projection scheme). In a second time substep, we solve the radiative part of the equations. This amounts to solving a differential equation where the source term is an integral operator. In the models we are interested in, the integral operator stems from the resolution of an elliptic equation. There are two possible approaches to discretize the radiative part : we can either try to solve the elliptic equation, or use a quadrature formula for the integral. We shall compare both methods.

Our main motivation is the computation of shock profiles and the checking of some theoretical results that were derived in $[18,9]$ for a scalar model, and in $[14,15]$ for a more complete model. Because shock profiles are asymptotically constant at $\pm \infty$, we shall see that it is reasonable to truncate the elliptic equation on the bounded domain where the solution is computed by forcing Dirichlet boundary conditions. If one is interested in solving the equations in a general situation, namely for a very general class of initial data, the nonlocal operator will raise some additional difficulties because it precludes the finite speed of propagation. Therefore, the choice of the computational domain will play a major role. Another motivation for this work is the behavior of large amplitude shock profiles. This is well understood for the scalar model studied in $[18,9]$, where it was shown that shock profiles of arbitrary strength are 
monotone functions. For the more complete model studied in $[14,15]$, there are no theoretical results and we want to check that shock profiles for this model capture the main features of radiative shocks, see e.g. $[16,21]$. In particular, we shall check numerically that large shock profiles exhibit a post-shock region where the temperature is non-monotone. This property was already displayed for other models in radiative hydrodynamics, see e.g. $[2,3]$ and $[16,21]$. The main point is that our method does not require any mesh refinement near the shock to exhibit these peaks in the temperature profile.

The paper is divided as follows. In section 2, we present the scalar toy model for radiating gases and develop two numerical methods. Our first method is based on the discretization of an elliptic equation, while the second method is based on a quadrature rule for the integral representation of the solution to this elliptic equation. In section 3, we introduce a more involved model that couples the Euler equations and an elliptic equation. We develop some numerical methods for this system that have some similarities with the methods introduced for the toy model. We illustrate both sections with some numerical experiments that confirm some earlier theoretical results.

\section{Numerical schemes for a toy model}

\subsection{The numerical schemes}

In this section, we propose a numerical procedure to solve the toy model :

$$
\left\{\begin{array}{l}
\partial_{t} u+\partial_{x}\left(u^{2} / 2\right)=q-u, \quad t \geq 0, x \in \mathbb{R}, \\
-\partial_{x x} q=u-q .
\end{array}\right.
$$

Observe that the elliptic equation for $q$ can be solved explicitly using a convolution :

$$
q(t, x)=\frac{1}{2} \int_{\mathbb{R}} \mathrm{e}^{-|x-y|} u(t, y) d y .
$$

However, it is not clear whether it is more efficient to use a quadrature formula for this integral representation rather than to discretize the elliptic equation. In what follows, we shall compare these two possible approaches. Both methods will satisfy the maximum principle at the discrete level. Recall that entropic solutions of (1) satisfy the maximum principle, see $[13,19]$ for a complete study of the Cauchy problem for (1) and of various asymptotic regimes.

Our main goal is to compute shock profiles, that are traveling waves $u(x-\sigma t)$ that connect two end states $u_{l}$ and $u_{r}$ :

$$
\lim _{\xi \rightarrow-\infty} u(\xi)=u_{l}, \quad \lim _{\xi \rightarrow+\infty} u(\xi)=u_{r}, \quad u_{l}>u_{r}, \quad \sigma=\frac{u_{r}+u_{l}}{2}
$$

The speed $\sigma$ of the traveling wave is given by the Rankine-Hugoniot conditions for the Burgers equation, and the inequality $u_{l}>u_{r}$ is the entropic condition. It has been shown in $[9,18]$ that such shock profiles exist for all $u_{l}>u_{r}$. (The traveling wave is even unique up to a shift.) However, the smoothness of the traveling wave depends on the amplitude $u_{l}-u_{r}$ of the shock. In particular, when $u_{l}-u_{r}>\sqrt{2}$, the shock profile is discontinuous at the origin (it is $C^{\infty}$ everywhere else). From the integral representation of $q$, we see that $q$ has the same limits as $u$ at $\pm \infty$ if $u$ is a shock profile. This fact will be helpful in our numerical scheme below.

The numerical procedure we propose is based on a splitting strategy. We first solve the convective term $\partial_{x}\left(u^{2} / 2\right)$ by means of the Godunov scheme. Then we solve the source terms $q-u$. Let us first introduce 
some notations. Let $L>0$ denote the half-length of the space interval where the numerical solution will be computed, and let $M$ denote the number of interior cells. The space step is $\Delta x=2 L /(M+2)$, and we let $\Delta t>0$ denote the time step. Eventually, we let $u_{j}^{n}$ denote the approximation of the entropic solution $u$ to (1) at time $t^{n}=n \Delta t, n \in \mathbb{N}$, and at the position $x_{j}=-L+2(j-1 / 2) L /(M+2), j \in\{1, \ldots, M+2\}$. Similarly, the approximation of $q$ is denoted $q_{j}^{n}$.

The definition of the numerical scheme is the following. We first initialize the numerical solution :

$$
\forall j \in\{1, \ldots, M+2\}, \quad u_{j}^{0}= \begin{cases}u_{l}, & \text { if } 2 j \leq M+2, \\ u_{r}, & \text { if } 2 j>M+2,\end{cases}
$$

where $u_{l, r}$ are the end states of the shock profile that we are going to compute. Assume now that the $u_{j}^{k}$ 's are known for all $j \in\{1, \ldots, M+2\}$, and all integer $k \in\{0, \ldots, n\}$. We first update the numerical solution by solving the convective terms and set :

$$
\forall j \in\{2, \ldots, M+1\}, \quad u_{j}^{n+1 / 2}=u_{j}^{n}-\frac{\Delta t}{\Delta x}\left(G\left(u_{j}^{n}, u_{j+1}^{n}\right)-G\left(u_{j-1}^{n}, u_{j}^{n}\right)\right),
$$

where $G$ is the Godunov flux for the Burgers equation :

$$
\forall(u, v) \in \mathbb{R}^{2}, \quad G(u, v)= \begin{cases}\max \left(u^{2} / 2, v^{2} / 2\right), & \text { if } u \geq v \\ \min _{w \in[u, v]} w^{2} / 2, & \text { if } u \leq v .\end{cases}
$$

The second and final step consists in solving the radiative part of (1). To this end, we can first compute the radiative density $\left(q_{j}^{n+1 / 2}\right)$ by solving the linear system :

$$
\left(\begin{array}{ccccc}
2+\Delta x^{2} & -1 & & & \\
-1 & 2+\Delta x^{2} & -1 & & \\
& \ddots & \ddots & \ddots & \\
& & -1 & 2+\Delta x^{2} & -1 \\
& & & -1 & 2+\Delta x^{2}
\end{array}\right)\left(\begin{array}{c}
q_{2}^{n+1 / 2} \\
\vdots \\
q_{M+1}^{n+1 / 2}
\end{array}\right)=\Delta x^{2}\left(\begin{array}{c}
u_{2}^{n+1 / 2} \\
u_{3}^{n+1 / 2} \\
\vdots \\
u_{M}^{n+1 / 2} \\
u_{M+1}^{n+1 / 2}
\end{array}\right)+\left(\begin{array}{c}
u_{l} \\
0 \\
\vdots \\
0 \\
u_{r}
\end{array}\right)
$$

In terms of the elliptic equation, this amounts to solving :

$$
q-\partial_{x x} q=u^{n+1 / 2}, \quad q(-L)=u_{l}, \quad q(L)=u_{r},
$$

by means of the classical finite difference approximation. Of course, the Dirichlet boundary conditions are meaningful only if $L$ is chosen large enough so that $q$ does not vary too much outside of the interval $[-L, L]$. The matrix of the linear system (5) is symmetric positive definite, so the linear system (5) defines the $q_{j}^{n+1 / 2}$ 's in a unique way.

Eventually, we update the numerical solution as follows :

$$
\forall j \in\{2, \ldots, M+1\}, \quad u_{j}^{n+1}=\mathrm{e}^{-\Delta t} u_{j}^{n+1 / 2}+\left(1-\mathrm{e}^{-\Delta t}\right) q_{j}^{n+1 / 2} .
$$

The end points $j=1, j=M+2$ are fixed in order to match the asymptotic conditions for the shock profile, that is $u_{1}^{n+1}=u_{l}$, and $u_{M+2}^{n+1}=u_{r}$. The formula (6) is nothing but Duhamel's formula for the ordinary differential equation $\partial_{t} u=q-u$, where $q$ is assumed to be independent of time (in our case, $q$ is the solution of the discretized elliptic equation with the source term $u^{n+1 / 2}$ and Dirichlet boundary conditions). 
The other possibility we consider is to compute the radiative density at time $(n+1 / 2) \Delta t$ by means of a quadrature formula, and to update the numerical solution accordingly. Observe that (2) can be written equivalently as :

$$
q(t, x)=\frac{1}{2} \int_{0}^{+\infty} \mathrm{e}^{-y}(u(t, x+y)+u(t, x-y)) d y .
$$

We can use the Gauss-Laguerre quadrature formula :

$$
q(t, x) \simeq \frac{1}{2} \sum_{i=1}^{K} \omega_{i}\left(u\left(t, x+y_{i}\right)+u\left(t, x-y_{i}\right)\right),
$$

where $K$ is a given integer, $y_{1}, \ldots, y_{K}$ are the (real and distinct) roots of the $K$-th Laguerre polynomial, and $\omega_{1}, \ldots, \omega_{K}$ satisfy (see e.g. $[1,17]$ ):

$$
\forall i=1, \ldots, K, \quad \omega_{i}>0, \quad \omega_{1}+\cdots+\omega_{K}=1 .
$$

The second possibility to compute the radiative energy is therefore to set :

$$
\forall j=2, \ldots, M+1, \quad \tilde{q}_{j}^{n+1 / 2}=\frac{1}{2} \sum_{i=1}^{K} \omega_{i}\left(u_{j+d_{i}}^{n+1 / 2}+u_{j-d_{i}}^{n+1 / 2}\right), \quad d_{i}=\left\lfloor\frac{y_{i}}{\Delta x}\right\rfloor .
$$

In (8), there might be some indices $j$ and $i$ such that $j+d_{i}$ exceeds $M+2$, or $j-d_{i}$ is less than 1 . In that case, we impose the values $u_{r}$ and $u_{l}$, which amounts to considering that the numerical solution is constant outside of the interval $[-L, L]$ :

$$
\begin{cases}u_{k}^{n+1 / 2}=u_{r} & \text { if } k \geq M+2 \\ u_{k}^{n+1 / 2}=u_{l} & \text { if } k \leq 1\end{cases}
$$

Then the numerical solution is updated as in (6), but this time with the radiative energy $\tilde{q}^{n+1 / 2}$ given by (8):

$$
\forall j \in\{2, \ldots, M+1\}, \quad u_{j}^{n+1}=\mathrm{e}^{-\Delta t} u_{j}^{n+1 / 2}+\left(1-\mathrm{e}^{-\Delta t}\right) \tilde{q}_{j}^{n+1 / 2} .
$$

This procedure can be seen as a parallel of the Dirichlet boundary conditions. As previously, we also let $u_{1}^{n+1}=u_{l}$, and $u_{M+2}^{n+1}=u_{r}$.

The following Lemma states that both schemes (3), (4), (5), (6) and (3), (4), (8), (9) are monotone, provided that the CFL condition is satisfied :

Lemma 1. Let $u_{l}>u_{r}$, and $v_{l}>v_{r}$ be two pairs of end states, and let $\left(u_{j}^{n}\right),\left(v_{j}^{n}\right)$ denote the corresponding numerical solutions produced by the scheme (3), (4), (5), (6). Assume that the classical CFL condition :

$$
\Delta t \max \left(\left|u_{l}\right|,\left|u_{r}\right|,\left|v_{l}\right|,\left|v_{r}\right|\right) \leq \Delta x
$$

is satisfied. Then if the inequalities $u_{l} \leq v_{l}$, and $u_{r} \leq v_{r}$ hold, we have $u_{j}^{n} \leq v_{j}^{n}$ for all $j$ and all $n$. The same property holds for the scheme (3), (4), (8), (9).

The proof follows from a basic induction argument using the monotonicity of the Godunov scheme (see e.g. [8]), the monotonicity properties of the matrix in (5), and the properties of the weights $\omega_{i}$ in (8). We skip the proof that is almost straightforward. (Observe that Lemma 1 would hold if we had considered any monotone scheme for the Burgers equation.)

An important consequence of Lemma 1 is the fact that both schemes introduced above satisfy the maximum principle. All the $u_{j}^{n}$ 's are valued in the interval $\left[u_{r}, u_{l}\right]$. (Use first $v_{l}=v_{r}=u_{l}$, then $v_{l}=v_{r}=u_{r}$ in Lemma 1.) Before showing some numerical tests, we make a few remarks. 
Remark 1. - The schemes introduced above can be relevant only in the case where the expected theoretical solution is approximately constant at the end points of the interval $[-L, L]$. Since we expect that the shock profile will be the large time asymptotics of the numerical solution, this requires that the shock is stationary $(\sigma=0)$. In that case, $L$ should be chosen sufficiently large so that the solution is indeed approximately constant near $L$ and $-L$. Since shock profiles converge exponentially fast towards their end states (see $[18,9])$, the numerical value of $L$ is not so large, see the numerical tests below. For nonstationary shocks, one should change the space coordinate in order to make the shock stationary, which amounts to adding a transport term $\sigma \partial_{x} u$ in (1). (The numerical flux $G$ should be changed accordingly.)

- We expect to obtain the shock profile as the large time asymptotics of the numerical solution. In order to obtain this convergence, the initial data should be a zero mass perturbation of the exact shock profile, see [9] (if the initial mass of the perturbation is nonzero, the numerical solution should converge to a translation of the shock profile so the asymptotic solution may get out from the reference frame $[-L, L])$. However, we know from [9] that the shock profile is symmetric with respect to the end states $u_{l}$ and $u_{r}$, that is :

$$
\forall \xi \in \mathbb{R}, \quad u(\xi)-\frac{u_{l}+u_{r}}{2}=\frac{u_{l}+u_{r}}{2}-u(-\xi) .
$$

Consequently, the initial condition (3) is a zero mass perturbation of the shock profile. Another possible initial condition for the numerical scheme is :

$$
\forall j \in\{1, \ldots, M+2\}, \quad u_{j}^{0}=\frac{u_{l}+u_{r}}{2}+\frac{u_{r}-u_{l}}{2} \tanh x_{j},
$$

which is supposed to be a smaller perturbation of the shock profile (the convergence towards a continuous shock profile should therefore be quicker).

- Even though we are looking for an asymptotic solution, we use a first order scheme since the equation is scalar and the computational effort is not so important. The numerical solution attains its asymptotic value rather quickly, as we shall see in the next paragraph.

In the following paragraph, we check the behavior of the scheme on some examples.

\section{$2.2 \quad$ Numerical examples}

We first implement the schemes (3), (4), (5), (6) and (3), (4), (8), (9) for a 'small' shock profile. In this example, we have $u_{l}=-u_{r}=1 / 2$. The shock is stationary and according to the results in [9], the associated shock profile is smooth (at least within $C^{2}(\mathbb{R})$ ). The scheme is initialized with the hyperbolic tangent profile as suggested in the preceding remark. According also to the results in [9], the continuous shock profile $u$ should satisfy the second order differential equation:

$$
\left(u-\frac{u_{l}+u_{r}}{2}\right) u^{\prime \prime}+\left(u^{\prime}\right)^{2}+u^{\prime}-\frac{1}{2}\left(u-u_{r}\right)\left(u-u_{l}\right)=0
$$

It is natural to check how our numerical solution satisfies the discretized version of this differential equation, with $u^{\prime}$ replaced by its second order approximation $\left(u_{j+1}-u_{j-1}\right) /(2 \Delta x)$, and $u^{\prime \prime}$ replaced by $\left(u_{j+1}+u_{j-1}-2 u_{j}\right) / \Delta x^{2}$. This gives a measure of how far from the true shock profile the numerical solution is. In figure 1 we plot the numerical solution obtained after running sufficiently many time iterations for both schemes (using either the discretization of the elliptic equation or the Gauss-Laguerre quadrature formula), and we plot the logarithm of the error in order to check that our solution is a reasonable approximate solution of the differential equation (10). The computations are run using the 

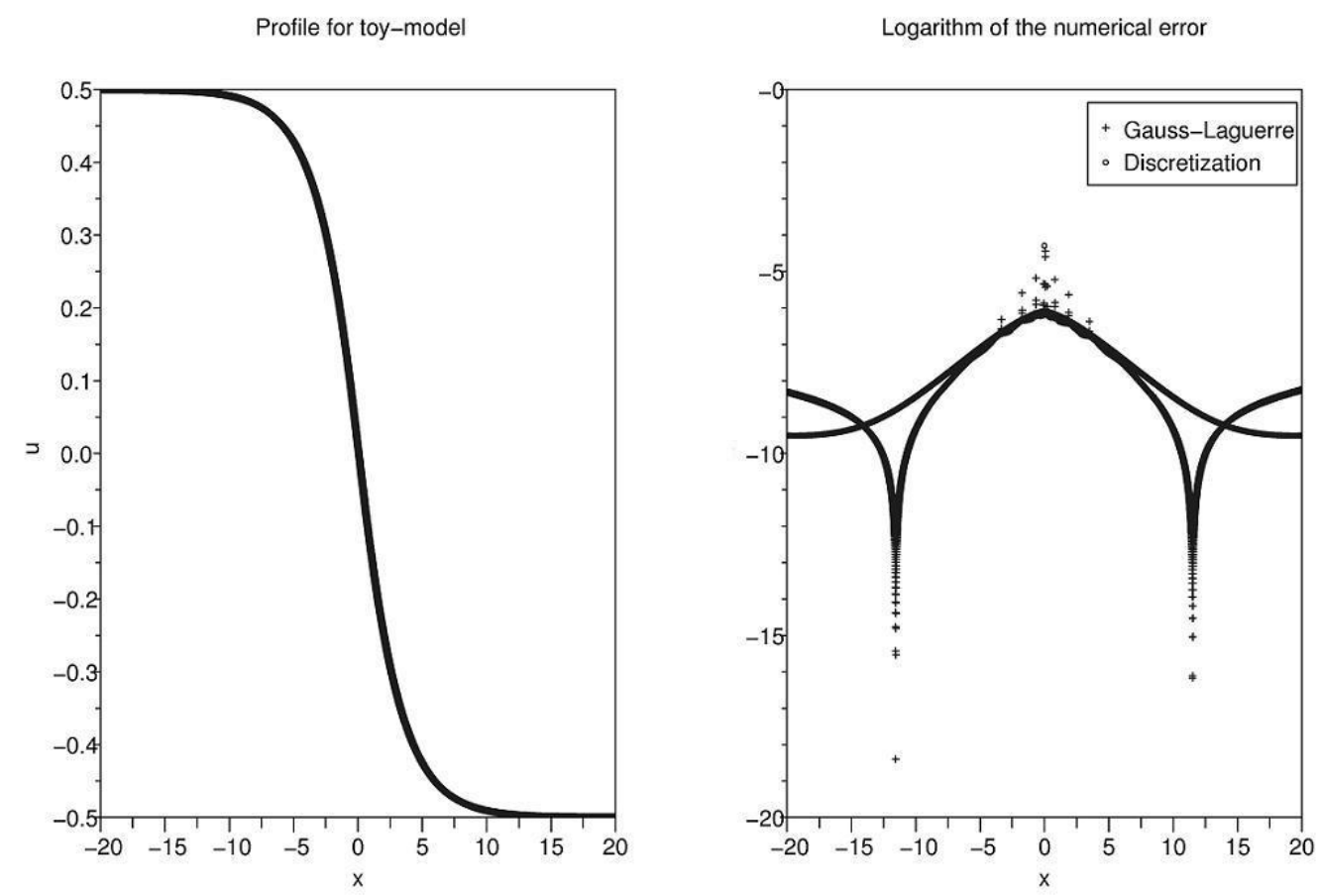

Figure 1: Shock profile for $u_{l}=-u_{r}=1 / 2$ (left), and logarithm of the numerical error (right).

parameters $L=20, M=10000, K=10$, and the number of time iterations is 5000 . The CFL condition is chosen in an optimal way. We refer to [1, chapter 29] for the numerical values of the $y_{i}, \omega_{i}$ 's.

The second example we deal with is $u_{l}=-u_{r}=2$. In this case, the associated shock profile is discontinuous. The main question to investigate is the number of points that the scheme produces in the discontinuity. As shown in figure 2, the results are very satisfactory. The parameters are still $L=20$, $M=10000, K=10$, and the number of time iterations is 5000 . The error is small away from the origin, that is in the region where the profile is smooth and (10) is supposed to be valid. At the origin, the conservation law (1) has to be understood in a weak sense and (10) does not hold anymore. In conclusion, our schemes capture in a very satisfactory way the shock profiles for (1).

Observe that as the amplitude of the shock increases, the convergence to the end states at $\pm \infty$ is quicker. This property is also predicted by the analysis in [9] where it appears that the convergence rate towards the end states is an increasing function of the amplitude. Observe also that when the quadrature formula is used to compute the radiative energy, the method is not able to capture accurately discontinuous shock profiles. The peaks in the error correspond to the points used in the quadrature formula. The reason for this misbehavior is that while the quadrature formula is exact for polynomials of degree less than $2 K-1$, the accuracy falls down for discontinuous functions. Using the discretization of the elliptic equation ensures a better smoothness for the numerical solution. This phenomenon pleads for using the discretization (5) over the quadrature formula (8), at least for this scalar model.

When the amplitude $u_{l}-u_{r}$ of the shock is very small, the (exponential) convergence rate of the shock profile towards its end states tends to 0 , see [9]. To compute such solutions we therefore need to consider 

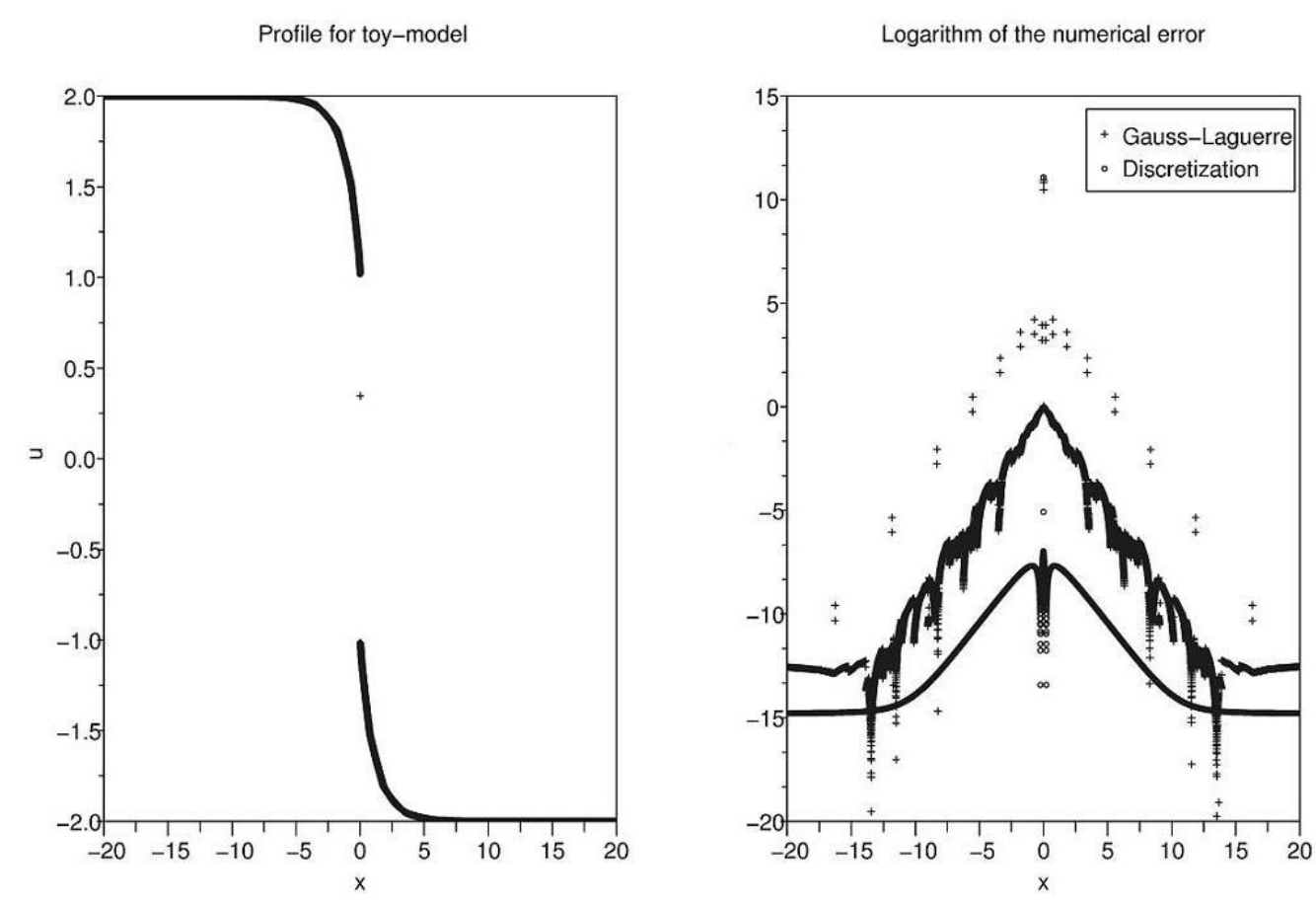

Figure 2: Shock profile for $u_{l}=-u_{r}=2$ (left), and logarithm of the numerical error (right).

a large value of $L$, which in turn leads to a large value of $M$ (because the space step should not be too large). The number of time iterations also increases.

\section{Numerical schemes for radiative hydrodynamics}

\subsection{The numerical schemes}

The aim of this section is to derive a numerical scheme to compute the shock profiles for the following model of radiative hydrodynamics (see [16]), written in a dimensionless form :

$$
\left\{\begin{array}{l}
\partial_{t} \rho+\partial_{x}(\rho u)=0, \\
\partial_{t}(\rho u)+\partial_{x}\left(\rho u^{2}+p\right)=0, \\
\partial_{t}(\rho E)+\partial_{x}(\rho E u+p u)=\sigma\left(q-\theta^{4}\right), \\
-\partial_{x x} q=\theta^{4}-q .
\end{array} \quad t \geq 0, x \in \mathbb{R}\right.
$$

In (11), $\rho$ is the density of the fluid, $u$ is the velocity, $p$ is the pressure, $E=e+u^{2} / 2$ is the total energy, $e$ is the internal energy, $\theta$ is the temperature, $q$ is the radiative energy and $\sigma$ is a positive constant. The equations are closed by assuming that the fluid obeys the perfect gas pressure law :

$$
p=R \rho \theta=(\gamma-1) \rho e,
$$

where $R$ is the perfect gas constant, and $\gamma>1$ is the ratio of specific heats at constant pressure and constant volume. In all what follows, we choose $\rho, u, \theta$ as the unknown functions in (11) and we consider 
$p, e$ as functions of $\rho, \theta$. The motivation for this choice comes from the expression of the source terms in (11). Shock profiles for (11) are traveling waves connecting some end states $\left(\rho_{l}, u_{l}, \theta_{l}\right)$ and $\left(\rho_{r}, u_{r}, \theta_{r}\right)$ that satisfy the Rankine-Hugoniot conditions for the standard Euler equations as well as the entropy inequality. When the amplitude of the shock is sufficiently small, smooth shock profiles have been shown to exist in [14] (see [12] for related results when the elliptic equations is linear). It is believed that shock waves of bigger amplitude admit a piecewise smooth shock profile, as for the toy model (1). However, this question has not yet received a rigorous treatment. The aim of this section is to check numerically the properties of smooth shock profiles, in particular the monotonicity of the main quantities (density, pressure, temperature) when the shock amplitude is small, but also to observe what should be the properties of shock profiles associated to a large shock wave. In most models of radiative hydrodynamics, see e.g. the classical textbooks $[21,16]$, the temperature profile experiments a post-shock peak and is non-monotone. We shall check numerically this behavior for our simple model (11).

We keep the same notations as in the preceding section for the computational domain $[-L, L]$, the space and time steps $\Delta t, \Delta x$. The approximations of $\rho, u \ldots$ are denoted $\rho_{j}^{n}, u_{j}^{n}$ and so on. Our numerical schemes for (11) are still based on a splitting strategy. Given some end states $\left(\rho_{l}, u_{l}, \theta_{l}\right)$ and $\left(\rho_{r}, u_{r}, \theta_{r}\right)$ that define a stationary shock wave solution to the Euler equations, we initialize the numerical solution as before :

$$
\forall j \in\{1, \ldots, M+2\}, \quad\left(\rho_{j}^{0}, u_{j}^{0}, \theta_{j}^{0}\right)= \begin{cases}\left(\rho_{l}, u_{l}, \theta_{l}\right), & \text { if } 2 j \leq M+2, \\ \left(\rho_{r}, u_{r}, \theta_{r}\right), & \text { if } 2 j>M+2 .\end{cases}
$$

Assuming that the numerical solution is known up to time $t^{n}=n \Delta t$, the time evolution is defined through the splitting formula :

$$
\left(\rho^{n+1}, u^{n+1}, \theta^{n+1}\right)=X^{\Delta t} Y^{\Delta t}\left(\rho^{n}, u^{n}, \theta^{n}\right),
$$

where $X^{\Delta t}$ denotes the numerical approximation at time $\Delta t$ of the solution of the differential equation :

$$
\left\{\begin{array}{l}
\dot{\rho}=0, \\
(\dot{\rho} u)=0, \\
(\dot{\rho} \dot{E})=\sigma\left(q-\theta^{4}\right), \\
-\partial_{x x} q=\theta^{4}-q,
\end{array}\right.
$$

and $Y^{\Delta t}$ denotes the numerical approximation at time $\Delta t$ of the solution of the standard Euler equations :

$$
\left\{\begin{array}{l}
\partial_{t} \rho+\partial_{x}(\rho u)=0 \\
\partial_{t}(\rho u)+\partial_{x}\left(\rho u^{2}+p\right)=0 \\
\partial_{t}(\rho E)+\partial_{x}(\rho E u+p u)=0
\end{array}\right.
$$

Below we are going to give the precise definition of the operators $X^{\Delta t}$ and $Y^{\Delta t}$. There are many numerical methods available for the Euler equations in the literature, so we shall mainly focus on the definition of the approximation $X^{\Delta t}$ of (12).

\subsubsection{Computation of the convective terms}

We shall use two different ways to solve numerically the Euler equations. This will enable us to compare the accuracy of both methods. The first method is the classical Godunov method, see e.g. [8] for an extensive description. If we write the Euler equations in the conservative form :

$$
\partial_{t} U+\partial_{x} f(U)=0, \quad U=(\rho, \rho u, \rho E)^{T},
$$


and if we start from some distribution $\left(U_{j}^{n}\right)_{1 \leq j \leq N+2}$, then Godunov's method reads :

$$
\forall j \in\{2, \ldots, M+1\}, \quad U_{j}^{n+1 / 2}=U_{j}^{n}-\frac{\Delta t}{\Delta x}\left(f\left(W\left(0, U_{j}, U_{j+1}\right)\right)-f\left(W\left(0, U_{j-1}, U_{j}\right)\right)\right),
$$

where $W(\xi, U, V)$ is the solution of the Riemann problem at $\xi=x / t$ with left and right states $U$ and $V$. Of course this definition coincides with (4) for Burgers' equation. Godunov's method is very precise but has a serious computational cost because it requires to solve a Riemann problem at each cell. For the Euler equations, this requires to find the intersection of wave curves, which is done numerically by a Newton type algorithm that can be found in [20, chapter 4.9].

The second method we shall use is the so-called Lagrange-projection method introduced in $[11,5,6,7$, 10,4]. This method is divided in two substeps. The first substep solves the Euler equations in Lagrangian coordinates by means of a finite volume approximation. In the second substep, the numerical solution is projected on the Eulerian grid by means of an antidiffusive procedure. We refer to the above mentionned references for the precise definition of the numerical fluxes.

\subsubsection{Computation of the radiative part}

Here we focus on the numerical resolution of the differential equation (12). Let an initial distribution $\left(\rho_{j}^{n+1 / 2}, u_{j}^{n+1 / 2}, \theta_{j}^{n+1 / 2}\right)_{1 \leq j \leq M+2}$ be given by the resolution of the convective part. We first reduce the differential equation (12) to the equivalent system:

$$
\left\{\begin{array}{l}
\dot{\rho}=\dot{u}=0, \\
\dot{\theta}=\frac{\sigma}{c_{v} \rho}\left(q-\theta^{4}\right), \\
-\partial_{x x} q=\theta^{4}-q,
\end{array}\right.
$$

where $c_{v}=R /(\gamma-1)$ is the specific heat at constant volume, and $\rho$ denotes the constant value of the density. It is not possible to use Duhamel's formula anylonger to solve (14) because the differential equation is not linear in the variable $\theta$. Therefore, we solve (14) by an implicit/semi-implicit method. The need of an implicit method is motivated by the fact that, if $\theta$ is larger than 1 , the source terms increase very rapidly, so that the differential equation gets stiffer and stiffer.

Our first numerical method for (14) is the following. Starting from $\left(\rho_{j}^{n+1 / 2}, u_{j}^{n+1 / 2}, \theta_{j}^{n+1 / 2}\right)$, we first compute the radiative energy by solving the elliptic equation by standard finite differences. More precisely, we first solve the linear system :

$$
\left(\begin{array}{ccccc}
2+\Delta x^{2} & -1 & & & \\
-1 & 2+\Delta x^{2} & -1 & & \\
& \ddots & \ddots & \ddots & \\
& & -1 & 2+\Delta x^{2} & -1 \\
& & & -1 & 2+\Delta x^{2}
\end{array}\right)\left(\begin{array}{c}
q_{2}^{n+1 / 2} \\
\vdots \\
q_{M+1}^{n+1 / 2}
\end{array}\right)=\Delta x^{2}\left(\begin{array}{c}
\left(\theta_{2}^{n+1 / 2}\right)^{4} \\
\vdots \\
\\
\left(\theta_{M+1}^{n+1 / 2}\right)^{4}
\end{array}\right)+\left(\begin{array}{c}
\theta_{l}^{4} \\
0 \\
\vdots \\
0 \\
\theta_{r}^{4}
\end{array}\right) .
$$

We can also compute the radiative energy through the Gauss-Laguerre quadrature rule as in the previous section. We omit the detailed definitions for the sake of simplicity.

Using this radiative energy $q^{n+1 / 2}$, we solve the ordinary differential equation:

$$
\dot{\theta_{j}}=\frac{\sigma}{c_{v} \rho_{j}^{n+1 / 2}}\left(q_{j}^{n+1 / 2}-\theta_{j}^{4}\right),
$$


on a time step $\Delta t$. To compute the solution of the ODE, we use an implicit Euler method, that ensures the stability : we then have to solve at each step the following polynomial equation:

$$
\frac{\theta_{j}^{n+1}-\theta_{j}^{n+1 / 2}}{\Delta t}=\frac{\sigma}{c_{v} \rho_{j}^{n+1 / 2}}\left(q_{j}^{n+1 / 2}-\left(\theta_{j}^{n+1}\right)^{4}\right),
$$

that amounts to solving the 4th degree equation:

$$
\theta^{4}+\frac{\theta}{\widetilde{\Delta t}}=y
$$

with $y=q_{j}^{n+1 / 2}+\theta_{j}^{n+1 / 2} / \widetilde{\Delta t}>0$ and $\widetilde{\Delta t}=\sigma \Delta t /\left(c_{v} \rho_{j}^{n+1 / 2}\right)$. Performing classical computations on this last equation, one finds that the only positive root of (16) reads:

$$
\theta=\frac{-\sqrt{2 p}+\sqrt{\frac{\sqrt{2}}{\widetilde{\Delta t} \sqrt{p}}-2 p}}{2}
$$

where:

$$
p=\sqrt[3]{\frac{1+\sqrt{1+\frac{256 \widetilde{\Delta t} y^{3}}{27}}}{16}}+\sqrt[3]{\frac{1-\sqrt{1+\frac{256 \widetilde{\Delta t} y^{3}}{27}}}{16}} .
$$

The 'numerical' approximation of the differential equation (12) at time $\Delta t$ is then defined as follows :

$$
X^{\Delta t}\left(\rho_{j}^{n+1 / 2}, u_{j}^{n+1 / 2}, \theta_{j}^{n+1 / 2}\right)_{1 \leq j \leq M+2}=\left(\rho_{j}^{n+1 / 2}, u_{j}^{n+1 / 2}, \theta_{j}^{n+1}\right)_{1 \leq j \leq M+2} .
$$

Remark 2. Using the same solver for the polynomial equation (16), we could have used a second-order resolution through the Crank-Nicolson scheme with $y=2 q_{j}^{n+1 / 2}-\left(\theta_{j}^{n+1 / 2}\right)^{4}+\theta_{j}^{n+1 / 2} \widetilde{\widetilde{\Delta t}}>0$ and $\widetilde{\Delta t}=$ $\sigma \Delta t /\left(2 c_{v} \rho_{j}^{n+1 / 2}\right)$.

Whereas, for the Euler implicit scheme, it is clear that the temperature $\theta^{n+1}$ belongs to the interval $\left[\min \left(\theta^{n+1 / 2}\right), \max \left(\theta^{n+1 / 2}\right)\right]$, this property is not straightforward anylonger for the Crank-Nicolson scheme. Furthermore, since we chose a scheme of order one for the Euler equations, it seems reasonable to stick to the fully implicit Euler scheme that is also of order 1.

\section{$3.2 \quad$ Numerical examples}

The stationary 1-shock waves for the Euler equations can be parametrized by the quantities $\rho_{l}>0$, $u_{l} \in \mathbb{R}, \beta \in(1,(\gamma+1) /(\gamma-1))$, see $[8]$ :

$$
\begin{aligned}
& \rho_{r}=\beta \rho_{l}, \quad u_{r}=\frac{u_{l}}{\beta}, \quad j=\rho_{l} u_{l}, \\
& p_{l}=j^{2} \frac{\gamma+1-\beta(\gamma-1)}{2 \gamma \rho_{r}}, \quad p_{r}=p_{l} \frac{(\gamma+1) \beta-(\gamma-1)}{\gamma+1-\beta(\gamma-1)}, \quad \theta_{l}=\frac{p_{l}}{R \rho_{l}}, \quad \theta_{r}=\frac{p_{r}}{R \rho_{r}} .
\end{aligned}
$$

For our numerical tests, we chose $R=1, \gamma=1.4$ (diatomic molecules) and $\sigma=1$ for simplicity. We first show in Figures 3, 5, 4, 6 the results for an expected smooth profile $\left(\rho_{l}=1, \beta=1.05, u_{l}=1.2\right.$, 
$M=1000, L=40$ and 1000 time iterations), then we show a discontinuous profile in Figures $7,9,8,10$ ( $\rho_{l}=1, \beta=3, u_{l}=3$ for $M=2000, L=40$ and 2000 time iterations). We have used the implicit Euler scheme to solve the differential equation (14). The results with the Crank-Nicolson scheme are similar.

As in the previous section, a measure of the relevance of the numerical simulation is given by the computations of the errors: remembering that we only consider stationary shocks, we focus here on

- how the conservation of mass and momentum is satisfied: observe that, in the continuous setting, the Rankine-Hugoniot condition implies that, $\rho u=\rho_{l} u_{l}=\rho_{r} u_{r}=: \mathscr{J}$ and $\rho u^{2}+p=\rho_{l}\left(u_{l}\right)^{2}+p_{l}=$ $\rho_{r}\left(u_{r}\right)^{2}+p_{r}=: \mathscr{J} C$,

- how the numerical velocity profile matches the underlying second-order differential equation:

$$
\left(u-\frac{\gamma C}{\gamma+1}\right) u^{\prime \prime}+\left(u^{\prime}\right)^{2}-\frac{4(\gamma-1)}{\mathscr{J}(\gamma+1) R^{4}}(C-u)^{3} u^{3}(C-2 u) u^{\prime}-\frac{1}{2}\left(u-u_{l}\right)\left(u-u_{r}\right)=0,
$$

where $\rho$ (resp. $u$ ) is the continuous profile of density (resp. velocity).

\subsection{Conclusion}

The numerical methods that we have developed here capture how smooth the temperature profile is for shocks of small amplitude. Note that the Lagrange-projection scheme is more accurate than Godunov's, and that when we use the Gauss-Laguerre rule to compute the radiative energy, the post-shock error is bigger than when we use the discretization method. This is particularly highlighted in the case of discontinuous profiles where we can see that the error produced by the discretization method decreases rapidly after the shock. Again, the Lagrange-projection method seems to be more accurate. Let us say in favor of Godunov's scheme that there are less points in the discontinuity.

We also noted that the post-shock peak in temperature seems to be going bigger when the amplitude $\beta$ of the shock increases. (More simulations are available at http://profilchoc.gforge.inria.fr/.)

Furthermore, despite what is announced in [16, p. 563], we have found no example of strong shocks that admit a smooth shock profile. The model (11) seems to present the same dichotomy as the toy model (1): there is a critical threshold for the shock amplitude under which the profile is continuous and above which the profile is discontinuous. Although we conducted a huge panel of numerical tests (varying $\beta$ for different values of $u_{l}$ ), we have not been able to deduce general properties concerning monotonicity nor to find numerical thresholds characterizing the loss of continuity. The main obstacle is the fact that the time needed to reach the asymptotic profile turns out to vary too much with the various parameters $\left(\rho_{l}\right.$, $\left.u_{l}, \beta\right)$.

Acknowledgements The authors would like to thank Frédéric Lagoutière and Magali Ribot for helpful discussions, and the INRIA staff in Lille for the Gforge collaborative environment. Experiments presented in this paper were carried out using the Grid'5000 experimental testbed, an initiative from the French Ministry of Research through the ACI GRID incentive action, INRIA, CNRS and RENATER and other contributing partners (see https:// www.grid5000.fr).

\section{References}

[1] M. Abramowitz, I. A. Stegun. Handbook of mathematical functions. Dover, 1992. 
[2] E. Audit, B. Dubroca, and J.-F. Ripoll. A factored operator method for solving coupled radiation hydrodynamics models. Transp. Theory Stat. Phys., 31:531-557, 2002.

[3] C. Buet, B. Després. Asymptotic preserving and positive schemes for radiation hydrodynamics. $J$. Comp. Phys., 215(2):717-740, 2006.

[4] B. Després and F. Lagoutière. Un schéma non linéaire anti-dissipatif pour l'équation d'advection linéaire. C. R. Acad. Sci. Paris Sér. I Math., 328(10):939-944, 1999.

[5] B. Després and F. Lagoutière. Contact discontinuity capturing schemes for linear advection and compressible gas dynamics. J. Sci. Comput., 16(4):479-524 (2002), 2001.

[6] B. Després and F. Lagoutière. Generalized Harten formalism and longitudinal variation diminishing schemes for linear advection on arbitrary grids. M2AN Math. Model. Numer. Anal., 35(6):1159-1183, 2001.

[7] B. Després and F. Lagoutière. A longitudinal variation diminishing estimate for linear advection on arbitrary grids. C. R. Acad. Sci. Paris Sér. I Math., 332(3):259-263, 2001.

[8] E. Godlewski, P.-A. Raviart. Numerical approximation of hyperbolic systems of conservation laws. Springer-Verlag, 1996.

[9] S. Kawashima, S. Nishibata. Shock waves for a model system of the radiating gas. SIAM J. Math. Anal., 30(1):95-117, 1998.

[10] F. Lagoutiere. Numerical resolution of scalar convex equations: explicit stability, entropy and convergence conditions. In CEMRACS 1999 (Orsay), volume 10 of ESAIM Proc., pages 183-199 (electronic). Soc. Math. Appl. Indust., Paris, 1999.

[11] F. Lagoutière. A non-dissipative entropic scheme for convex scalar equations via discontinuous cellreconstruction. C. R. Math. Acad. Sci. Paris, 338(7):549-554, 2004.

[12] C. Lattanzio, C. Mascia, and D. Serre. Shock waves for radiative hyperbolic-elliptic systems. Indiana Univ. Math. J., 56(5):2601-2640, 2007.

[13] C. Lattanzio, P. Marcati. Global well-posedness and relaxation limits of a model for radiating gas. J. Diff. Eq., 190(2):439-465, 2003.

[14] C. Lin, J.-F. Coulombel, and T. Goudon. Shock profiles for non-equilibrium radiating gases. Phys. $D, 218(1): 83-94,2006$.

[15] C. Lin, J.-F. Coulombel, and T. Goudon. Asymptotic stability of shock profiles in radiative hydrodynamics. Preprint, 2007.

[16] D. Mihalas, B. Weibel-Mihalas. Foundations of radiation hydrodynamics. Oxford University Press, 1984.

[17] M. Schatzman. Numerical analysis, a mathematical introduction. Oxford University Press, 2002.

[18] S. Schochet, E. Tadmor. The regularized Chapman-Enskog expansion for scalar conservation laws. Arch. Ration. Mech. Anal., 119(2):95-107, 1992. 
[19] D. Serre. $L^{1}$ stability of constants in a model for radiating gases. Comm. Math. Sci., 1(1):197-205, 2003.

[20] E. F. Toro. Riemann solvers and numerical methods for fluid dynamics. Springer-Verlag, second edition, 1999.

[21] Ya. B. Zeldovich, Yu. P. Raizer. Physics of shock waves and high temperature phenomena. Academic Press, 1966.
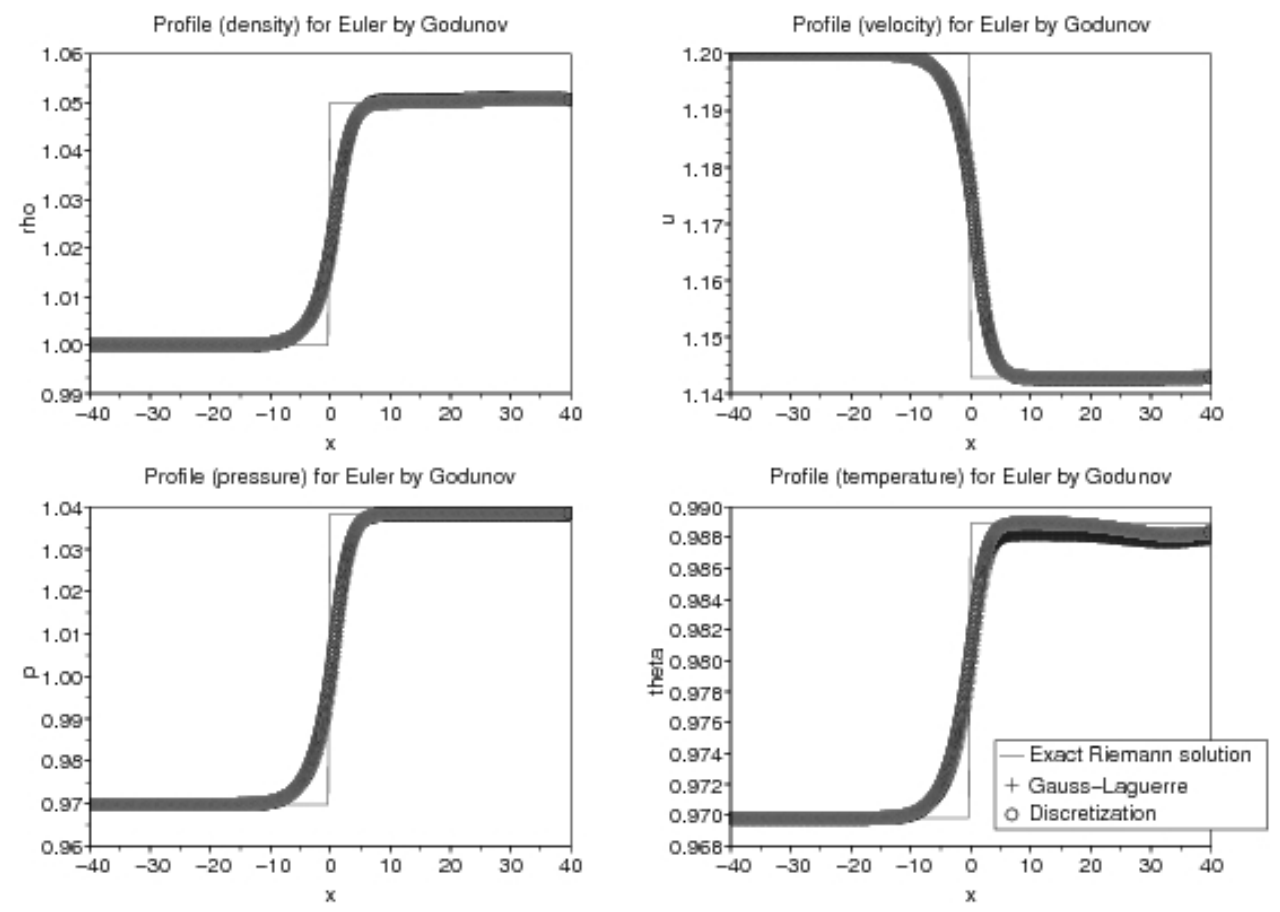

Figure 3: Shock profile obtained by Godunov's scheme. 

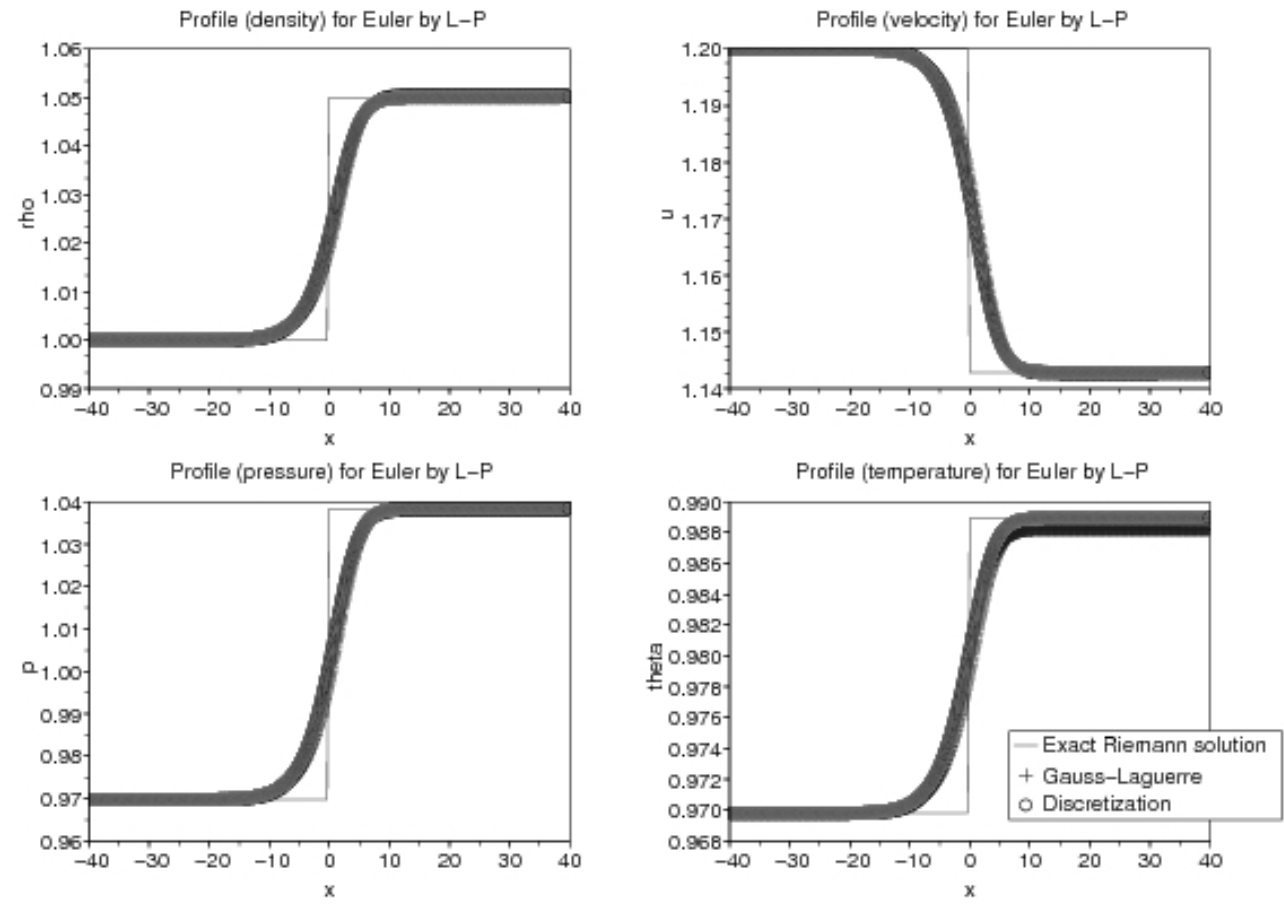

Figure 4: Shock profile obtained by the Lagrange-projection scheme.
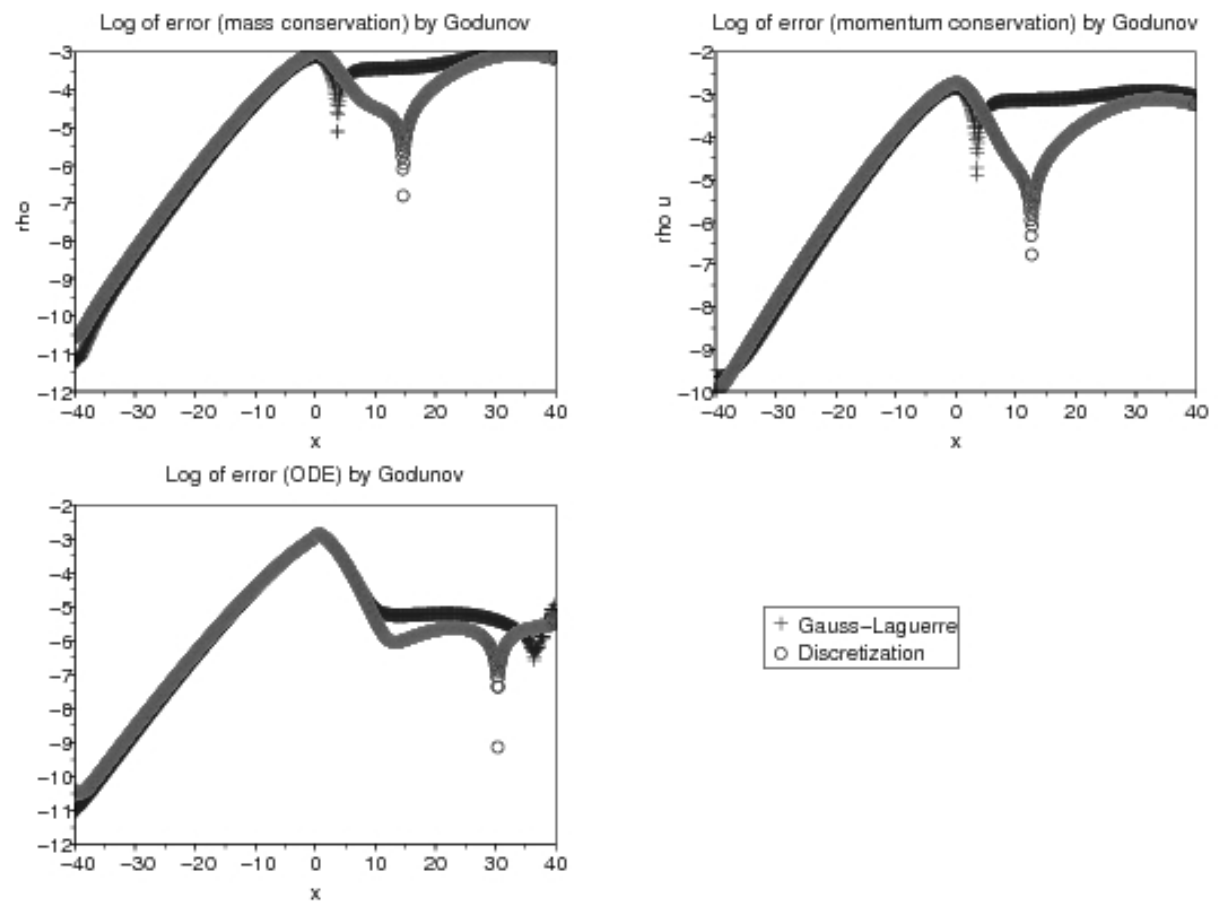

+ Gauss-Laguerre - Discretization

Figure 5: Error obtained by Godunov's scheme. 

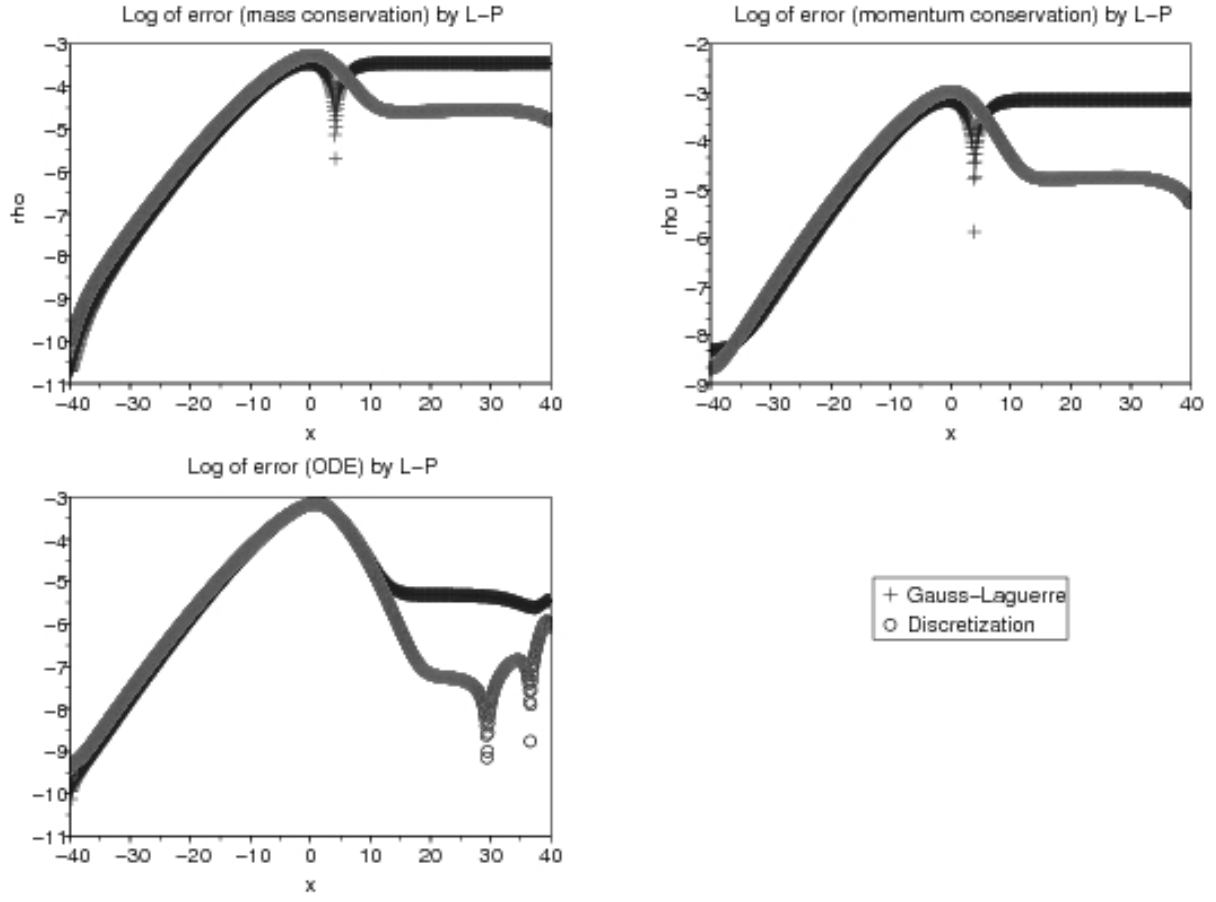

+ Gauss-Laguerre - Discretization

Figure 6: Error obtained by the Lagrange-projection scheme.
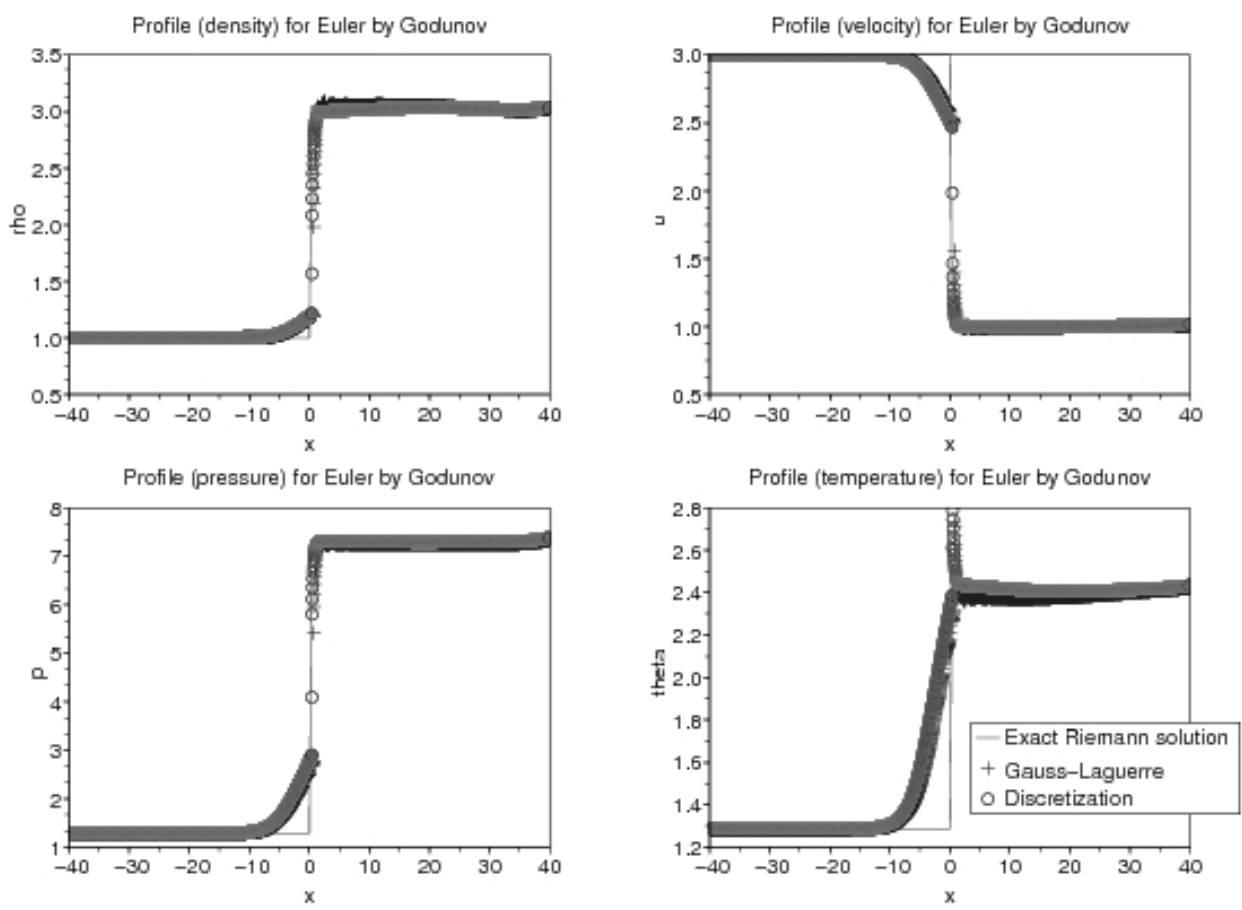

Figure 7: Shock profile obtained by Godunov's scheme. 

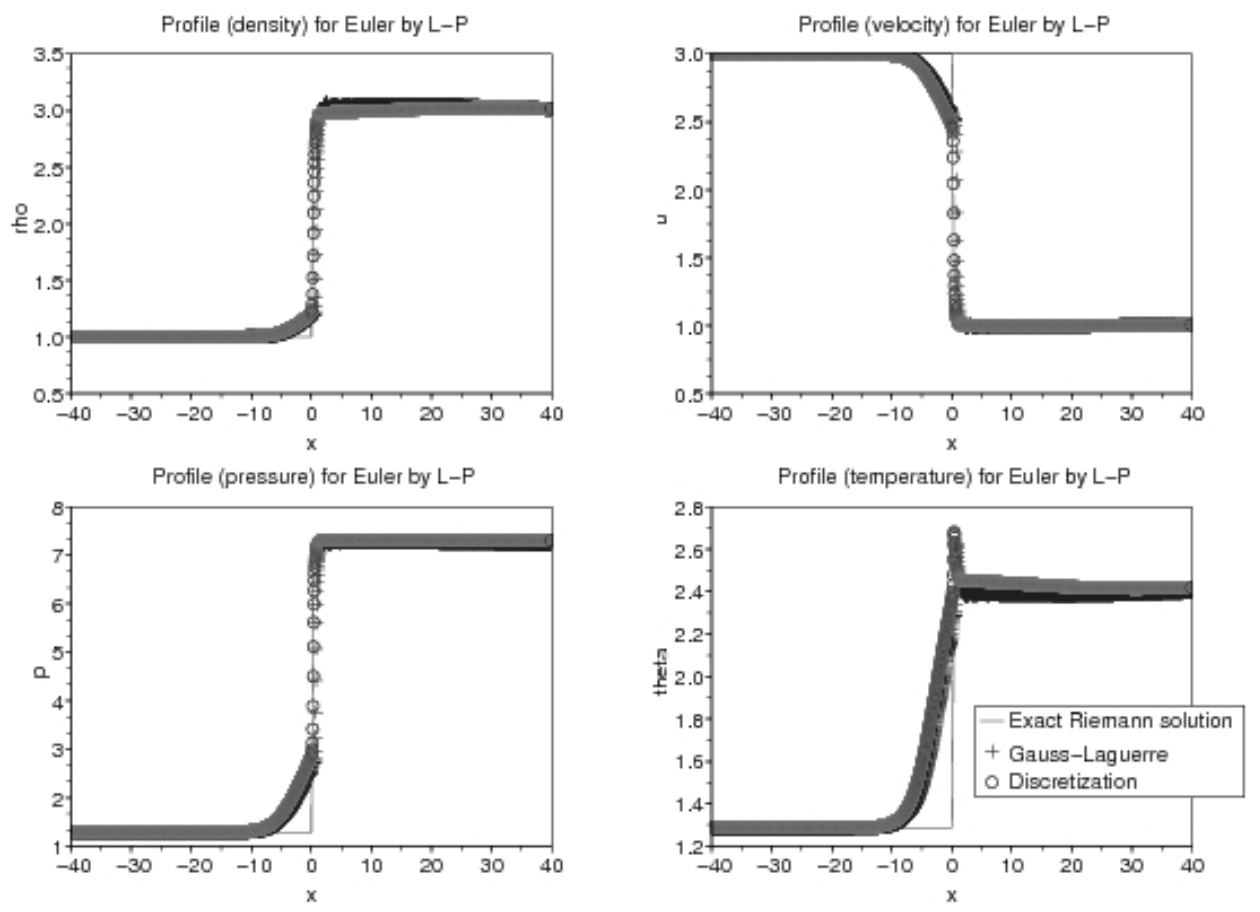

Figure 8: Shock profile obtained by the Lagrange-projection scheme.
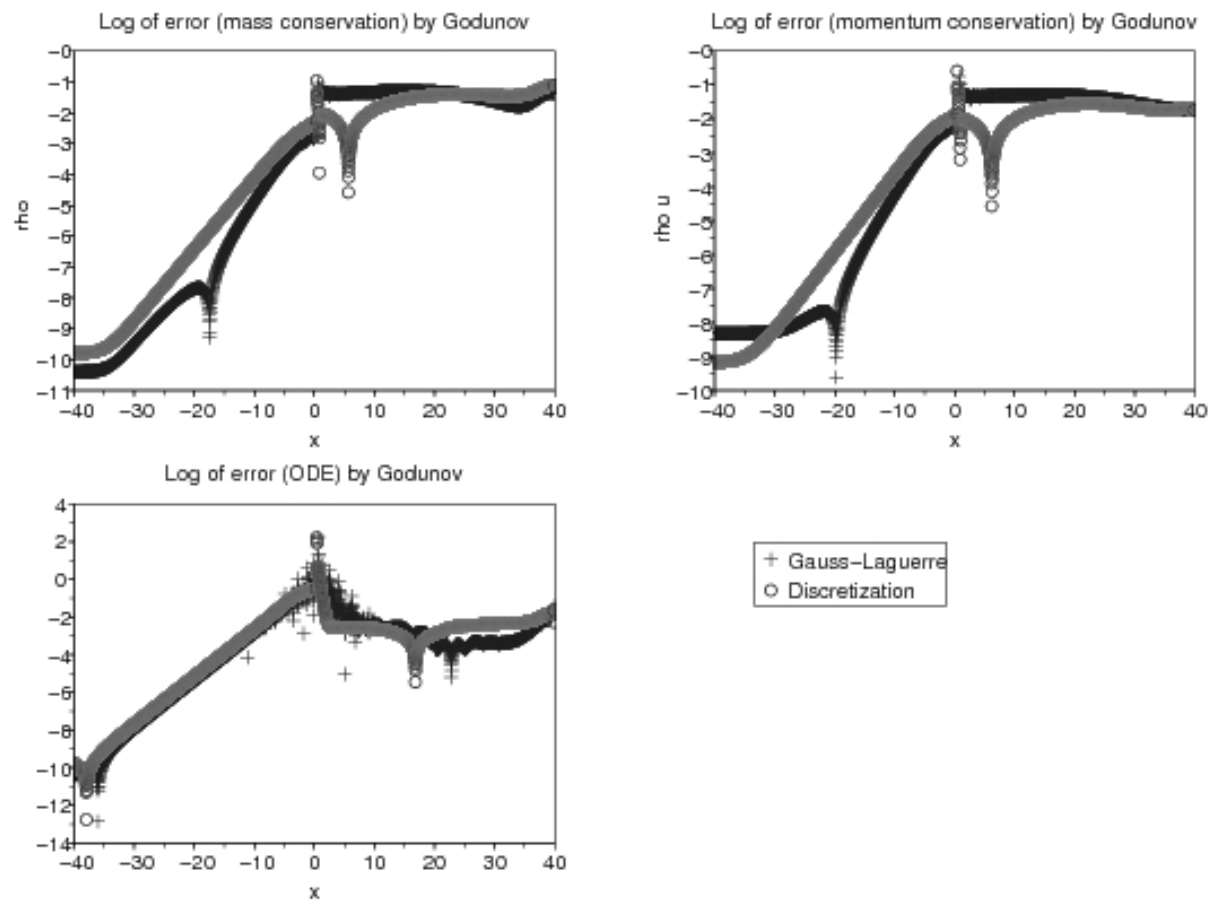

+ Gauss-Laguerr
- Discretization

Figure 9: Error obtained by Godunov's scheme. 

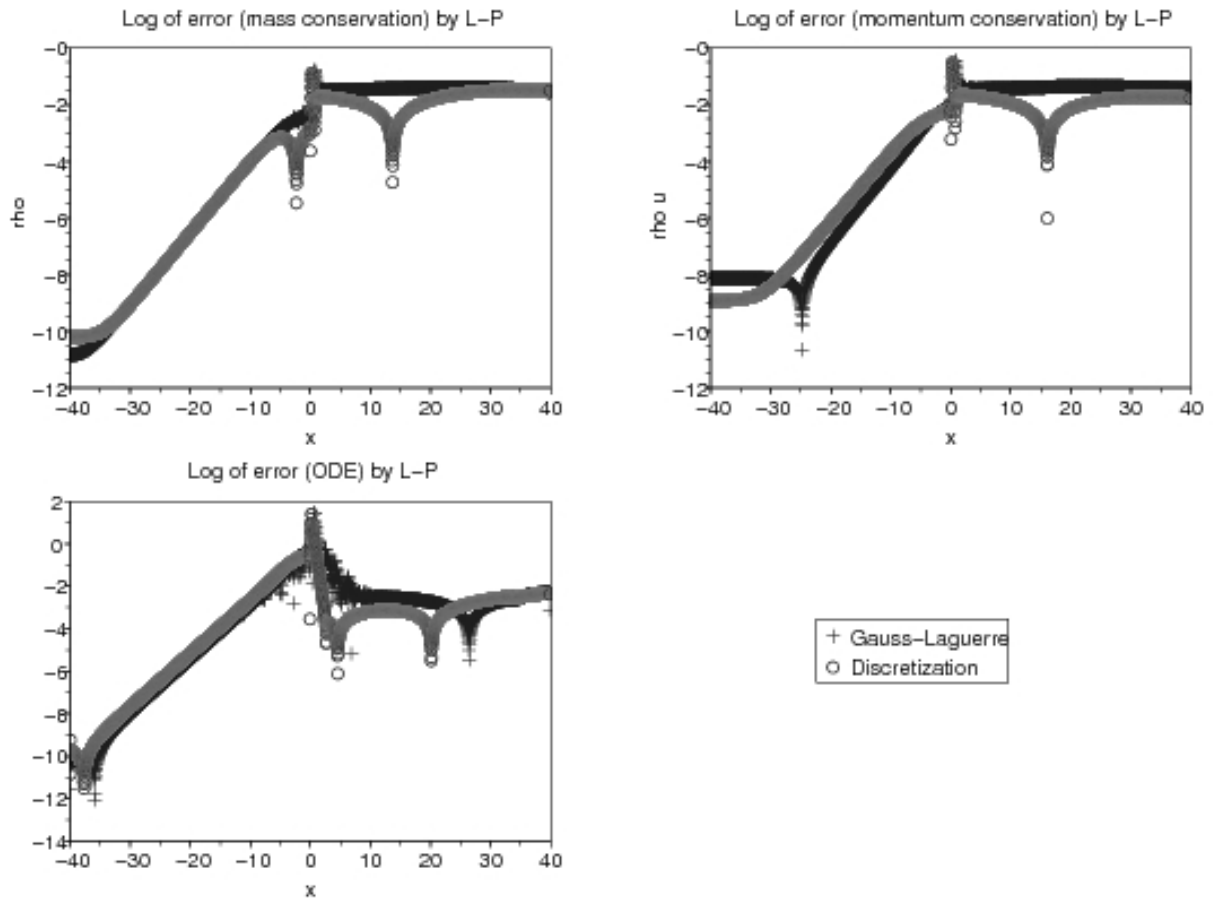

+ Gauss-Laguerre o Discretization

Figure 10: Error obtained by the Lagrange-projection scheme. 